\title{
Treatment with the PI3K inhibitor buparlisib (NVP-BKM120) suppresses the growth of established patient-derived GBM xenografts and prolongs survival in nude rats
}

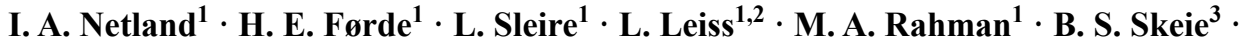 \\ H. Miletic ${ }^{4,5} \cdot$ P. Ø. Enger ${ }^{1,4,6} \cdot$ D. Goplen ${ }^{4,7}$
}

Received: 23 November 2015 / Accepted: 1 June 2016 / Published online: 9 June 2016

(C) The Author(s) 2016. This article is published with open access at Springerlink.com

\begin{abstract}
Glioblastomas (GBMs) are aggressive brain tumours with a dismal prognosis, despite combined surgery, radio- and chemotherapy. Close to $90 \%$ of all GBMs harbour a deregulated PI3K pathway, which is essential in regulating central cellular functions such as proliferation, cell growth, motility and survival. Thus, PI3K represents a potential target for molecular therapy in GBM. We investigated the anti-tumour efficacy of the PI3K inhibitor buparlisib (NVP-BKM120) in GBM cell lines in vitro and in vivo, when treatment was initiated after MRIconfirmed tumour engraftment. We found that buparlisib
\end{abstract}

I. A. Netland and H. E. Førde have contributed equally.

Electronic supplementary material The online version of this article (doi:10.1007/s11060-016-2158-1) contains supplementary material, which is available to authorized users.

D. Goplen

dorota@goplen.net

1 Oncomatrix Research Lab, Department of Biomedicine, University of Bergen, Bergen, Norway

2 Neuro Clinic, Haukeland University Hospital, Bergen, Norway

3 Department of Clinical Medicine, K1, University of Bergen, Bergen, Norway

4 Department of Biomedicine, Kristian Gerhard Jebsen Brain Tumour Research Center, University of Bergen, Bergen, Norway

5 Department of Pathology, Haukeland University Hospital, Bergen, Norway

6 Department of Neurosurgery, Haukeland University Hospital, Bergen, Norway

7 Department of Oncology, Haukeland University Hospital, Bergen, Norway inhibited glioma cell proliferation in a dose dependent manner, demonstrated by MTS assay, manual cell count and BrdU incorporation. A dose dependent increase in apoptosis was observed through flow cytometric analysis. Furthermore, by immunocytochemistry and western blot, we found a dose dependent inhibition of Akt phosphorylation. Moreover, buparlisib prolonged survival of nude rats harboring human GBM xenografts in three independent studies and reduced the tumours' volumetric increase, as determined by MRI. In addition, histological analyses of xenograft rat brains showed necrotic areas and change in tumour cell nuclei in buparlisib-treated animals. The rats receiving buparlisib maintained their weight, activity level and food- and water intake. In conclusion, buparlisib effectively inhibits glioma cell proliferation in vitro and growth of human GBM xenografts in nude rats. Moreover, the compound is well tolerated when administered at doses providing anti-tumour efficacy. Thus, buparlisib may have a future role in glioma therapy, and further studies are warranted to validate this compound for human use.

Keywords Glioblastoma - Brain tumours · PI3K · Proliferation - Targeted therapy $\cdot$ Patient-derived xenograft

\section{Introduction}

Glioblastoma (GBM) is the most aggressive primary brain tumour [1] with a median survival of 14.6 months. Less than $10 \%$ of patients survive 5 years after diagnosis [2]. The dismal prognosis for GBM patients, despite multimodal treatment calls for new therapeutic strategies.

Phosphatidylinositol 3-kinases (PI3Ks) are lipid kinases involved in cell proliferation, growth, apoptosis, DNA 
repair, angiogenesis, differentiation, motility and survival [3]. PI3Ks act as intermediate signaling molecules, involved in cell signaling pathways such as the PI3K/Akt/mTOR [4]. In normal cells, the PI3K activity is tightly regulated. Deregulation of the PI3K signaling pathway is common in cancer, including GBM [5]. Brennan and colleagues reported mutations in PI3K in $25.1 \%$ of GBMs, whereas $89.6 \%$ of GBMs had at least one alteration in the PI3K pathway including its other components, such as RTKs and PTEN [6]. Activation of this pathway is associated with increasing tumour grade and decreasing overall survival in gliomas [7]. The initial step in the PI3K pathway is PI3K-mediated phosphorylation of membrane bound phosphatidylinositols, generating second messengers (PIP3, PI 3,4-bisphosphate), which subsequently trigger a signaling cascade eventually activating multiple effector kinase pathways, such as the mTOR, ERK1/2, p38 MAPK, NF-kappa-B, and JNK/SAPK [4]. A key event in this cascade is binding of PIP3 to Akt, which is activated by phosphorylation at S473 and T308 [8]. The activation of Akt is assumed to be responsible for its growthpromoting and anti-apoptotic effects in tumour cells [4].

Given its role as a major regulator of multiple aspects of tumour cell behavior, PI3K has become a major target for drug design. Several small molecule PI3K inhibitors have been developed and are currently under pre-clinical and clinical assessment [4]. However, since the blood-brain-barrier (BBB) limits availability of drugs to the central nervous system (CNS), brain tumours are generally not considered attractive malignancies for initial drug screenings. For the same reason, few animal studies and hardly any patient trials have been published regarding the anti-tumour efficacy of PI3K inhibitors in gliomas.

Buparlisib (NVP-BKM120) is an orally bioavailable, small molecule compound with potent, pan-class I PI3K inhibitory capability against the $\mathrm{p} 110-\alpha,-\beta,-\delta$, and $-\gamma$ catalytic subunit isoforms at IC50 doses in a micromolar range [9]. Since buparlisib penetrates the BBB [9], it represents an attractive candidate for targeted glioma therapy. Previously, Koul et al. reported inhibition of cell line derived brain tumour growth in SCID mice using buparlisib administered 4 days following implantation of the tumour cells. In the clinical setting, therapy is usually initiated after the tumour is confirmed by MRI and biopsy. Here we demonstrate efficacy of buparlisib in treatment of patient derived in vivo passaged GBM biopsy material in the experimental setting resembling the usual clinical situation with tumour confirmed by MRI. The in vivo passaged GBM biopsies develop tumours that histologically closely resemble the GBM in situ [10]. The aim of the present study was to evaluate the efficacy of buparlisib treatment, initiated after confirming tumour engraftment by MRI in a clinically relevant brain tumour animal model [10].

\section{Materials and methods}

\section{Cell line and culturing}

Cells were maintained in humid incubators at $37^{\circ} \mathrm{C}$ and $5 \% \mathrm{CO}_{2}$. The U87 cell line was purchased from American Type Culture Collection (ATCC, Manassas, VA, USA) and cultured in DMEM (Sigma-Aldrich, St. Louis, MO, USA) supplemented with $10 \%$ fetal bovine serum, $3.2 \%$ nonessential amino acids, 100 units/mL Penicillin/Streptomycin, $400 \mathrm{~mol} / \mathrm{L}$ L-glutamine (all Lonza, Cologne, Germany) and $0.005 \mathrm{mg} / \mathrm{mL}$ Plasmocin (InvivoGen, San Diego, CA, USA). Prior to animal implantation, spheroids of U87 cells were prepared; 1000 cells were centrifuged at $2250 \mathrm{rpm}$ for $30 \mathrm{~min}$ in 96-well plates with conical bottom, containing $0.05 \%$ methylcellulose. After 7 days incubation, each spheroid contained 17,000 cells. Three spheroids $(51,000$ cells) were implanted in each animal. For in vitro assessment of buparlisib efficacy, a $10 \mathrm{mM}$ stock solution was prepared by dissolving buparlisib [kindly provided by Novartis (Basel, Switzerland)] in 100\% DMSO (Sigma-Aldrich).

\section{Patient tumour material and culturing}

Tumour biopsy tissue was obtained from the operating theatre, Haukeland University Hospital, Bergen, after approval from the regional Ethical Board and consent from patients. Xenograft spheroids (P3) were prepared from serially passaged GBM biopsy tissue (as described by Wang [10]) and cultured as described by Bjerkvig [11]. Prior to animal implantation, the spheroid material was enzymatically dissociated at $37^{\circ} \mathrm{C}$ by trypsin-EDTA and DNase (Roche, Basel, Switzerland) and resuspended in sterile PBS with $25 \mathrm{mM}$ glucose (both Sigma-Aldrich). 100,000 cells were implanted in each animal. For in vitro experiments, the spheroid material was grown in monolayer and maintained in NB medium (Thermo Fisher Scientific Corporation, Carlsbad, CA, USA) with the addition of $32 \mathrm{IE} / \mathrm{mL}$ heparin, $20 \mathrm{ng} / \mathrm{mL}$ bFGF and $20 \mathrm{ng} / \mathrm{mL}$ EGF (Millipore Corporation, Billerica, MA, USA).

\section{Cell viability (MTS assay)}

Cells were seeded in 96-well plates $24 \mathrm{~h}$ prior to treatment with buparlisib at concentrations ranging from 0 to $10 \mu \mathrm{M}$. After $72 \mathrm{~h}$ of exposure, the cells were subjected to MTS viability assay according to the manufacturer's protocol (CellTiter $96^{\circledR} \mathrm{AQ}_{\text {ueous }}$ One Solution Cell Proliferation Assay, Promega, Madison, WI, USA). Dose response curves for determination of IC50 values were generated in GraphPad Prism 6 (GraphPad Software Inc., La Jolla, CA, USA). 


\section{Cell count}

Cells were seeded $24 \mathrm{~h}$ prior to exposure of buparlisib for $72 \mathrm{~h}$, before enzymatically detached by Trypsin-EDTA solution (Sigma-Aldrich), and manually counted in a Burker chamber haemocytometer.

\section{BrdU-pulsing}

Cells exposed to buparlisib for $72 \mathrm{~h}$ were treated with $10 \mu \mathrm{M}$ BrdU (Sigma-Aldrich) for $45 \mathrm{~min}$ at $37^{\circ} \mathrm{C}$. They were detached using a cell scraper, washed with $1 \times$ PBS and resuspended to $1 \times 10^{5}$ cells $/ \mathrm{mL}$. $100 \mu \mathrm{L}$ cell suspension was loaded into individual sample chambers and centrifuged in a Shandon CytoSpin centrifuge (Thermo Fisher Scientific, Wilmington, DE, US) at $800 \mathrm{rpm}$ for $3 \mathrm{~min}$. Immobilized cells were fixed (ICC-section) and subsequently subjected to immunocytochemistry, imaging and quantification. For each slide, three randomly picked areas $\left(832 \times 665.6 \mu \mathrm{M}, 554 \mathrm{mM}^{2}\right)$ were selected for quantification. FITC stained cells and total number of cells was manually counted, and the proportion of FITC positive cells was calculated.

\section{Annexin V/propidium iodide (PI) apoptosis assay}

Cells were stained with the Annexin V apoptosis assay according to the manufacturer's protocol (Thermo Fisher Scientific). Samples were analysed on Accuri C6 (BD Biosciences) flow cytometer.

\section{Immunocytochemistry (ICC)}

Cells were fixed in $4 \%$ paraformaldehyde (Thermo Fisher Scientific Corporation) for $10 \mathrm{~min}$, permeabilized by $0.5 \%$ Triton X-100 (Sigma-Aldrich) in PBS for 4 min and incubated with blocking buffer [0.5\% BSA (Sigma-Aldrich) in PBS] for $15 \mathrm{~min}$. Cells were incubated with primary antibodies $\mathrm{O} / \mathrm{N}$ at $4{ }^{\circ} \mathrm{C}$. The primary antibodies used were total Akt, pAkt S473, pAkt T308 (all Cell Signaling Technology, Danvers, MA, USA), and BrdU (Abcam, Cambridge, UK) together with DNase (Roche). Following incubation, cells were washed in PBS and incubated with secondary antibodies for $45 \mathrm{~min}$ at $37^{\circ} \mathrm{C}$. The secondary antibodies used were FITC-conjugated [S473; goat anti-rabbit (Southern Biotechnologies Associates Inc., Birmingham, AL, USA)] or AF555-conjuagted [Total Akt; goat anti-mouse (Thermo Fisher Scientific)]. Cells were mounted with Vectashield mounting medium with DAPI (Vector Laboratories, Burlingame, CA, USA). Fluorescent images were obtained with a Nikon TE2000-E microscope (Nikon Corporation, Tokyo, Japan).

\section{Immunoblotting (western blot)}

Cells and tissue were harvested in kinexus buffer [20 mM MOPS, $5 \mathrm{mM}$ EDTA, $2 \mathrm{mM}$ EGTA, protease and phosphatase inhibitor tablets (Roche)], followed by sonication $3 \times 5$ s. Protein concentrations were determined by Pierce BCA Protein Assay Kit (Thermo Fisher Scientific Corporation). $20 \mu \mathrm{g}$ sample was mixed with LDS sample loading buffer and sample reducing agent (both NuPAGE, Thermo Fisher Scientific Corporation) and incubated at $70^{\circ} \mathrm{C}$ for $10 \mathrm{~min}$. Samples were run on a pre-cast NuPAGE SDS-gel (Thermo Fisher Scientific Corporation) at $200 \mathrm{~V}$ for $60 \mathrm{~min}$. Transfer to a nitrocellulose membrane was done at $30 \mathrm{~V}$ for $80 \mathrm{~min}$. Following blocking in 5\% (w/w) Difco Skim milk powder (Becton, Dickinson and Company, Franklin Lakes, NJ, USA), in TBST for $1 \mathrm{~h}$ at RT, the membranes were incubated with primary antibody (total Akt, pAkt S473, pAkt T308) and $\beta$-actin (Santa Cruz Biotechnology Inc, Dallas, TX, USA) or GAPDH (Abcam) at $4{ }^{\circ} \mathrm{C} \mathrm{O} / \mathrm{N}$. After washing, membranes were incubated with secondary antibodies [goat anti-mouse IgG-HRP (Santa Cruz Biotechnology Inc) and goat anti-Rabbit IgG $(\mathrm{H}+\mathrm{L})$ Cross Adsorbed Secondary Antibody, HRP conjugate (Thermo Fisher Scientific Corporation)] for $1.5 \mathrm{~h}$ at RT. For detection, Supersignal West Femto Maximum Sensitivity Substrate (Pierce Biotechnology, Rockford, IL, USA) was used, and chemiluminescent detection was obtained by a Fuji LAS 3000 Imager (Fuji Photo Film, Tokyo, Japan). Densitometric quantification was determined using ImageJ software (National Institutes of Health, Bethesda, MA, USA).

\section{Immunohistochemistry (IHC)}

Paraffin-embedded sections from P3 xenograft rat brains were deparaffinised using xylene, 100 and $96 \%$ ethanol, followed by antigen retrieval at $98^{\circ} \mathrm{C}$ for $25 \mathrm{~min}$ in $10 \mathrm{mM}$ citrate buffer, $\mathrm{pH}$ 6.0. Sections were treated with peroxidase and protein block (both Dako, Glostrup, Denmark) for 5 and $30 \mathrm{~min}$, respectively. The primary antibody mouse anti-nestin (Millipore Corporation) was diluted to 1:1000 in Tris-BSA buffer, and added to the slides for $1 \mathrm{~h}$ at room temperature. Following washing with TBS-Tween, sections were incubated with anti-mouse secondary antibody (Dako) for $45 \mathrm{~min}$ at room temperature. Slides were developed with DAB chromogen (Dako) and counterstained with hematoxylin.

\section{Animals}

Homozygous nude rats (rnu/rnu, Rowett) were used for the experiments. The animals were fed a standard pellet diet and provided water ad libitum, and kept in a pathogen free 
environment at a constant temperature and humidity with standard 12/12 h light and dark cycle. The experiment was approved by the Norwegian Animal Research Authority (Bergen, Norway). All animals were anaesthetized with $3 \%$ isoflurane gas (Abbott Laboratories, Abbot Park, IL, USA) mixed with $50 \%$ air and $50 \% \mathrm{O}_{2}$, and Marcaine (AstraZeneca, London, England) subcutaneously. Tumour implantation was performed as previously described [10]. Three animal studies were performed, and treatment started immediately after confirmed tumour take by MRI (U87 speroids; 10 days, P3 cell suspension; 21 days). Animals were randomly assigned to two different groups: (1) untreated controls and (2) $5 \mathrm{mg} / \mathrm{kg}$ buparlisib treatment (recommended by Novartis). Buparlisib suspension was prepared in $0.5 \%$ methyl cellulose and $0.5 \%$ Tween 20 (both SigmaAldrich), while control group received vehicle only $(0.5 \%$ methyl cellulose and $0.5 \%$ Tween 20 ). Both groups were treated 5 days a week and received $10 \mathrm{~mL} / \mathrm{kg}$ solution by oral gavage, using malleable oral dosing needles (Scanbur, Karlslunde, Denmark). Animals were weighed five times a week, inspected daily and euthanized by $\mathrm{CO}_{2}$ inhalation at the onset of symptoms.

\section{Magnetic resonance imaging (MRI)}

MRI scans of all animal brains were obtained after tumour implantation to assess the tumour growth using a Bruker Pharmascan 7T small animal MRI (Bruker Biospin MRI GmnH, Ettingen, Germany). Axial T1- and T2-weighted images were obtained as previously described [10]. The tumour volumes at treatment and follow-up MRI were calculated in Gamma Plan (Elekta Instrument AB, Stockholm, Sweden).

\section{Statistical analysis}

In vitro experiments were repeated three times and assessed by ANOVA with Tukey's multiple comparions test, with a $p$ value $<0.05$ considered significant. Kaplan-Meier survival curves were generated in GraphPad Prism 6 (GraphPad Software Inc.) for statistical analysis of the animal experiments. Median survival times for the treatment groups were compared using the log-rank test.

\section{Results}

\section{Buparlisib inhibits cell growth and induces apoptosis of GBM cells}

The cytotoxic potential of buparlisib on GBM cells was determined by exposing P3 GBM xenograft cells and U87 cells to buparlisib for $72 \mathrm{~h}$, and assessing viability using the
MTS assay. IC50 doses were found to be 1.17 and $0.84 \mu \mathrm{M}$ for U87 and P3 cells, respectively (Fig. 1a). To test the side effects of the drug on non-tumor cells, glial cells from NOD/SCID mice brains were sorted and cultured in vitro, subsequently treated with 1 or $10 \mu \mathrm{M}$ of buparlisib. After $72 \mathrm{~h}$ of exposure, treated cells and controls were analysed using the MTS assay. These data demonstrated that the metabolic activity of normal brain cells was significantly less affected than the P3 and U87 glioma cells (Supplementary Fig. 1). Furthermore, manual cell counting (Fig. 1b) showed that buparlisib reduced cell numbers in a dose dependent manner for both U87 and P3. In order to determine whether this reduction reflected increased cell death, decreased proliferation or both, untreated and buparlisib-treated cells were pulsed with the S-phase marker BrdU. Quantification of BrdU positive cells revealed a dose dependent decrease in cell proliferation (Fig. 1c), suggesting an anti-proliferate effect of buparlisib. The solvent (DMSO) did not show anti-proliferative effect (data not shown). Interestingly, flow cytometry of PI/Annexin V-stained cells demonstrated a dose-dependent increasing apoptosis upon buparlisib-treatment (Fig. 1d).

\section{Buparlisib inhibits phosphorylation of Akt in vitro}

The inhibitory effect of buparlisib on PI3K was shown by decreased activating phosphorylation of the PI3K downstream effector Akt. Akt is activated by phosphorylation of the amino acid residues threonine 308 (T308) and serine 473 (S473). ICC of U87 cells on coverslips, fixated after exposure of buparlisib for $72 \mathrm{~h}$ demonstrated a dose dependent reduction of Akt phosphorylation (Fig. 2a). Quantitative analysis was performed by western blot of lysates from U87 (Fig. 2b) and P3 (Fig. 2c) cells exposed to buparlisib in various concentrations and band intensities were assessed by densitometry. A dose dependent reduction of Akt phosphorylation at both sites (S473 and T308) was observed. Treatment with buparlisib did not alter the total level of Akt protein, indicating that the reduced level of phosphorylated Akt was caused by an inhibition of its phosphorylation and not by decrease of the Akt protein level.

\section{Buparlisib reduces tumour growth and prolongs survival in nude rats harbouring GBM xenografts}

The anti-tumour efficacy of buparlisib in vivo was evaluated in a clinically relevant GBM animal model that uses intracerebrally engrafted in vivo passaged GBM xenografts derived from human biopsy material. This model reflects the growth pattern of human tumours in situ, including infiltration into the brain parenchyma and angiogenesis [10]. Three weeks after implantation of the tumour, MRI confirmed tumour take, and the animals were randomly assigned to 
Fig. 1 a IC50 doses of buparlisib for P3 (left) and U87 (right) glioma cells. b Relative cell number of P3 (left) and U87 (right) glioma cells exposed to buparlisib for $72 \mathrm{~h}$. c Quantification of BrdU positive P3 (left) and U87 (right) glioma cells treated with buparlisib for $72 \mathrm{~h}$ with doses as indicated, and subsequently pulsed with BrdU. d Quantification of Annexin $\mathrm{V}$ - and PI-positive P3 (left) and U87 (right) glioma cells treated with buparlisib for $72 \mathrm{~h}$ with doses as indicated, and subsequently incubated with PI and Annexin V Alexa Fluor 488 conjugate. Error bars represent SEM. Red bars indicate $\mathrm{p}$ values for linear trends. All experiments were performed three times. $* \mathrm{p}<0.05, * * \mathrm{p}<0.01$, $* * * \mathrm{p}<0.001$
(A)

P3

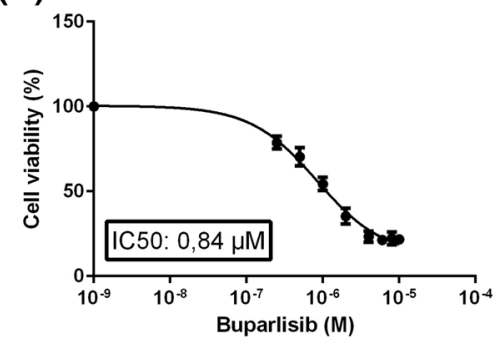

(B)

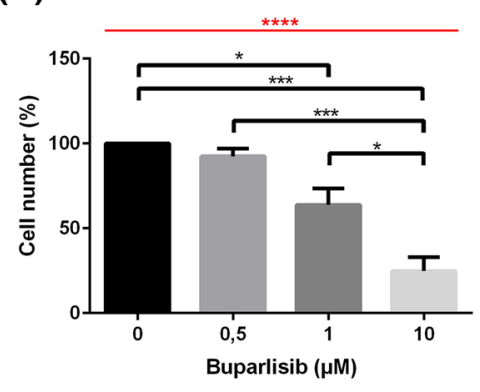

(C)

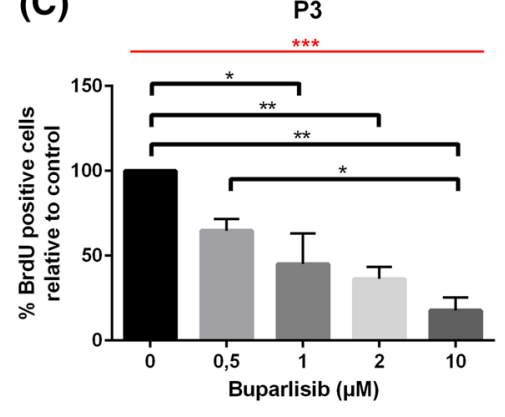

(D)

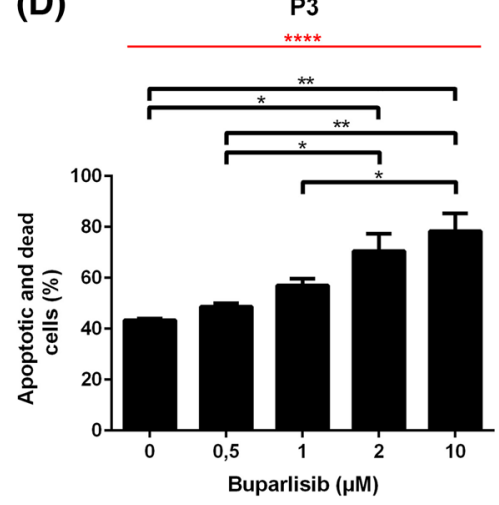

U87
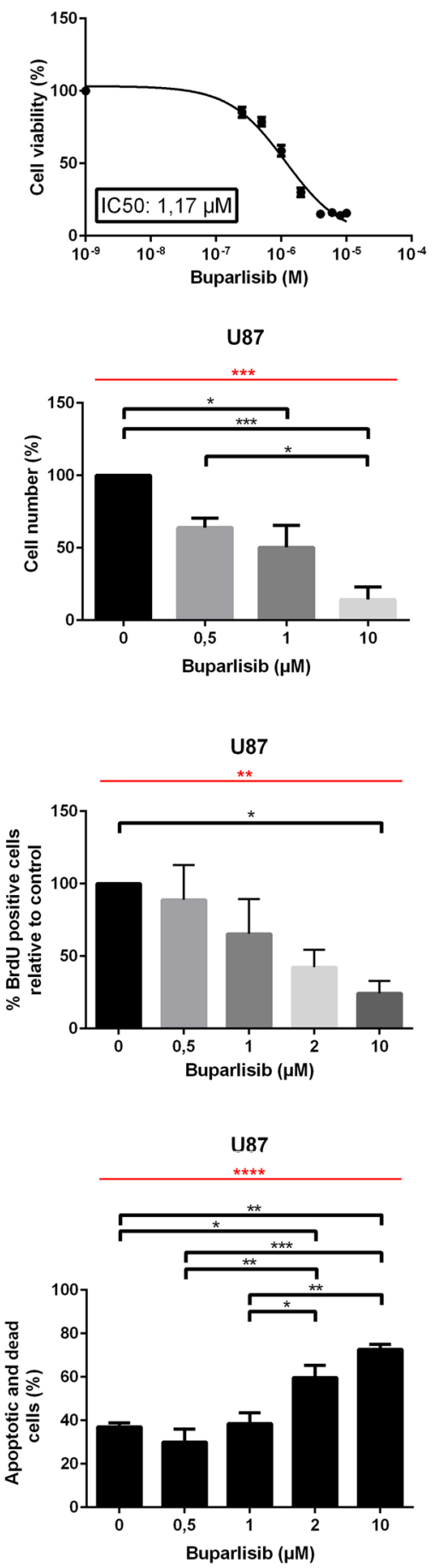

two treatment groups: one receiving $5 \mathrm{mg} / \mathrm{kg}$ buparlisib for 5 consecutive days and 2 days rest, and one group receiving vehicle only (control).

In the first study, the median survival from implantation was 36 days (range 31-40 days) for the buparlisib-treated rats $(n=5)$, and 30 days (range 29-35 days) for the control rats $(n=4)(p=0.039)$ (Fig. 3a). The survival benefit was confirmed in a second study with a median survival from tumour implantation of 51 days (range 50-54 days, $\mathrm{n}=3$ ) and 45 days (range 43-46 days, $\mathrm{n}=3$ ) in the treatment and control group respectively ( $p=0.0246$, Fig. $3 b$ ). MRI 2 weeks after treatment initiation revealed significantly smaller tumour volumes in the treatment group $\left[20.2 \mathrm{mM}^{2}\right.$ versus $103.4 \mathrm{mM}^{2}$ for the control group (Fig. 3c)]. Histology showed necrotic tumour areas in all four animals analysed from the treated group, while only one out of four animals from the control group showed a small necrotic tumour area (Fig. 3d). Furthermore, the density of tumour 

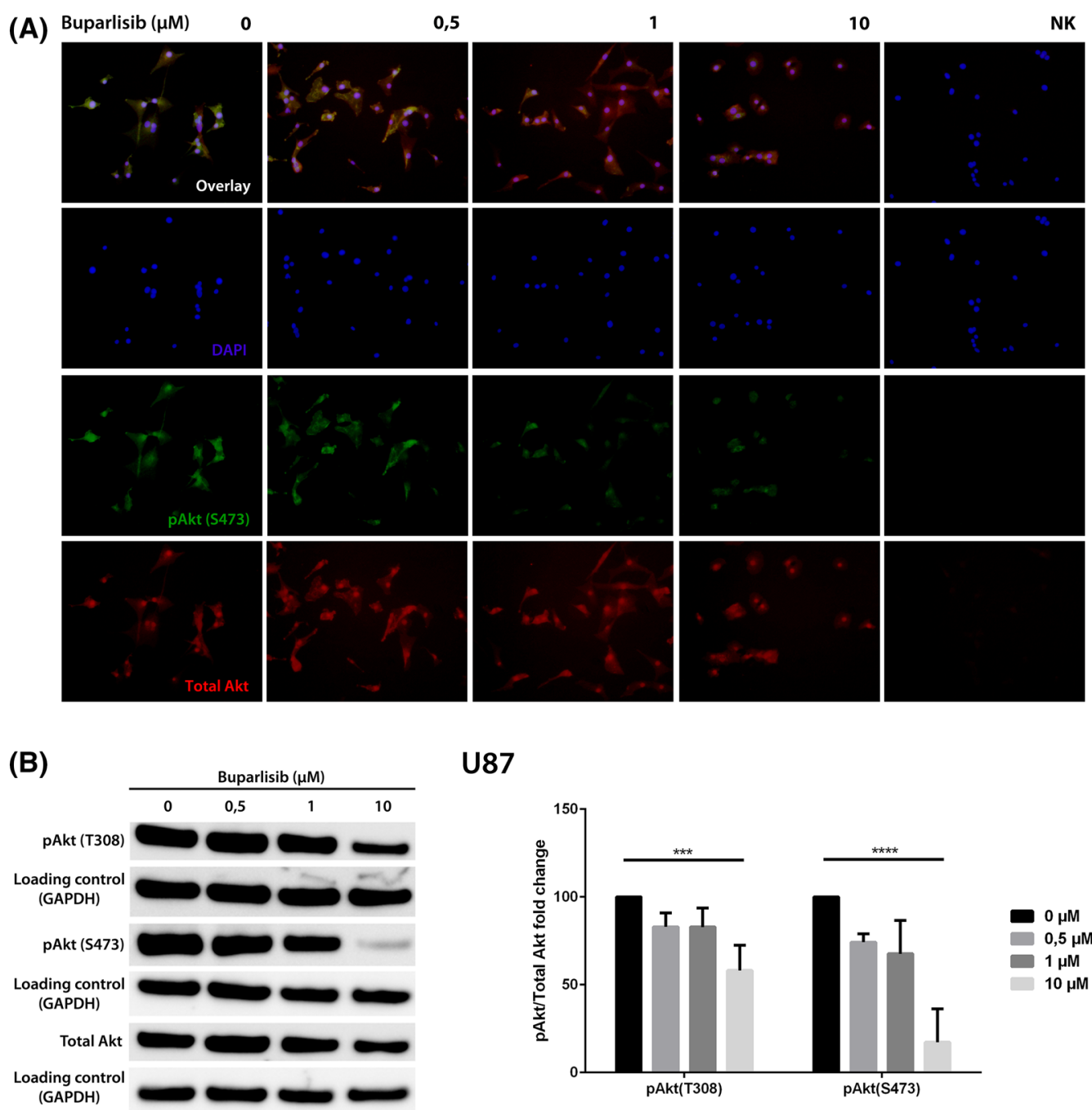

U87

(C)

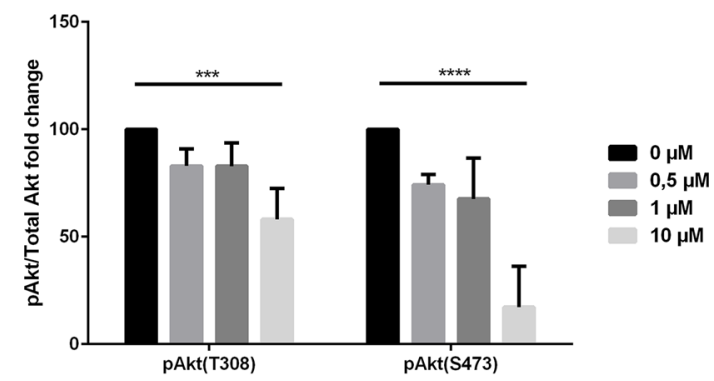

P3
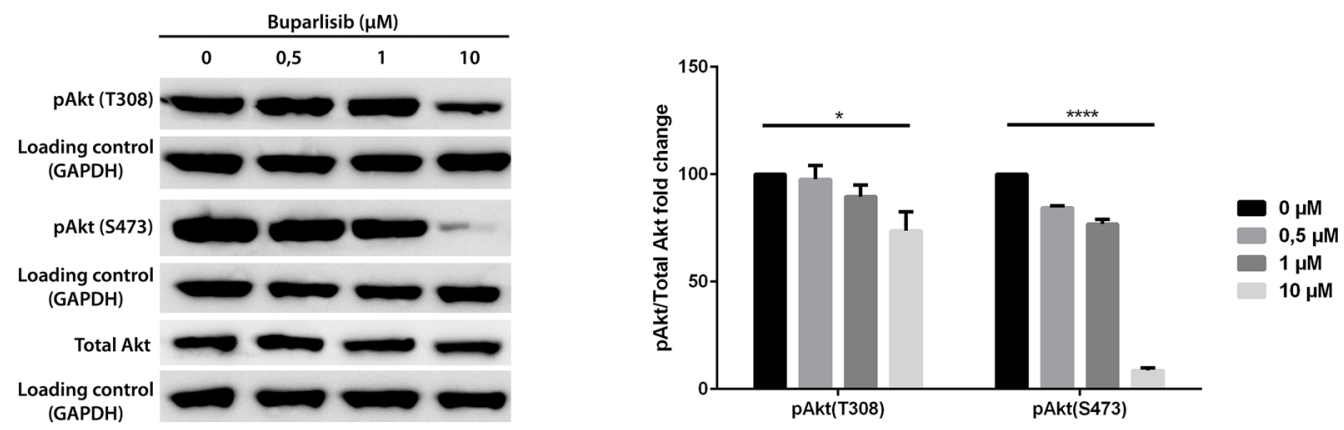

Fig. 2 a Immunocytochemistry showing Akt phosphorylation in U87cells at S473 after exposure to different concentrations of buparlisib for $72 \mathrm{~h}$. Upper panel overlay image of Akt phosphorylated at site S473 (FITC, green) and total Akt (AP555, red) with DAPI nuclear counterstaining (blue). Middle panel 1 DAPI nuclear staining (blue). Middle panel 2 Akt phosphorylated at site S473 (FITC, green). Lower panel total Akt-levels (AP555, red). bLeft western blots showing levels of pAkt (T308), pAkt (S473) and total Akt in U87 cells exposed

to buparlisib for $72 \mathrm{~h}$. Right densitometric assessment of western blots, showing relative changes in phosphorylation. cLeft western blots showing levels of pAkt (T308), pAkt (S473) and total Akt in P3 cells exposed to buparlisib for $72 \mathrm{~h}$. Right densitometric assessment of western blots, showing relative changes in phosphorylation. Error bars represent SEM of three independent experiments. $p$ values estimated represent linear trends. ${ }^{*} \mathrm{p}<0.05,{ }^{* *} \mathrm{p}<0.01,{ }^{* * *} \mathrm{p}<0.001$, $* * * * \mathrm{p}<0.0001$ 

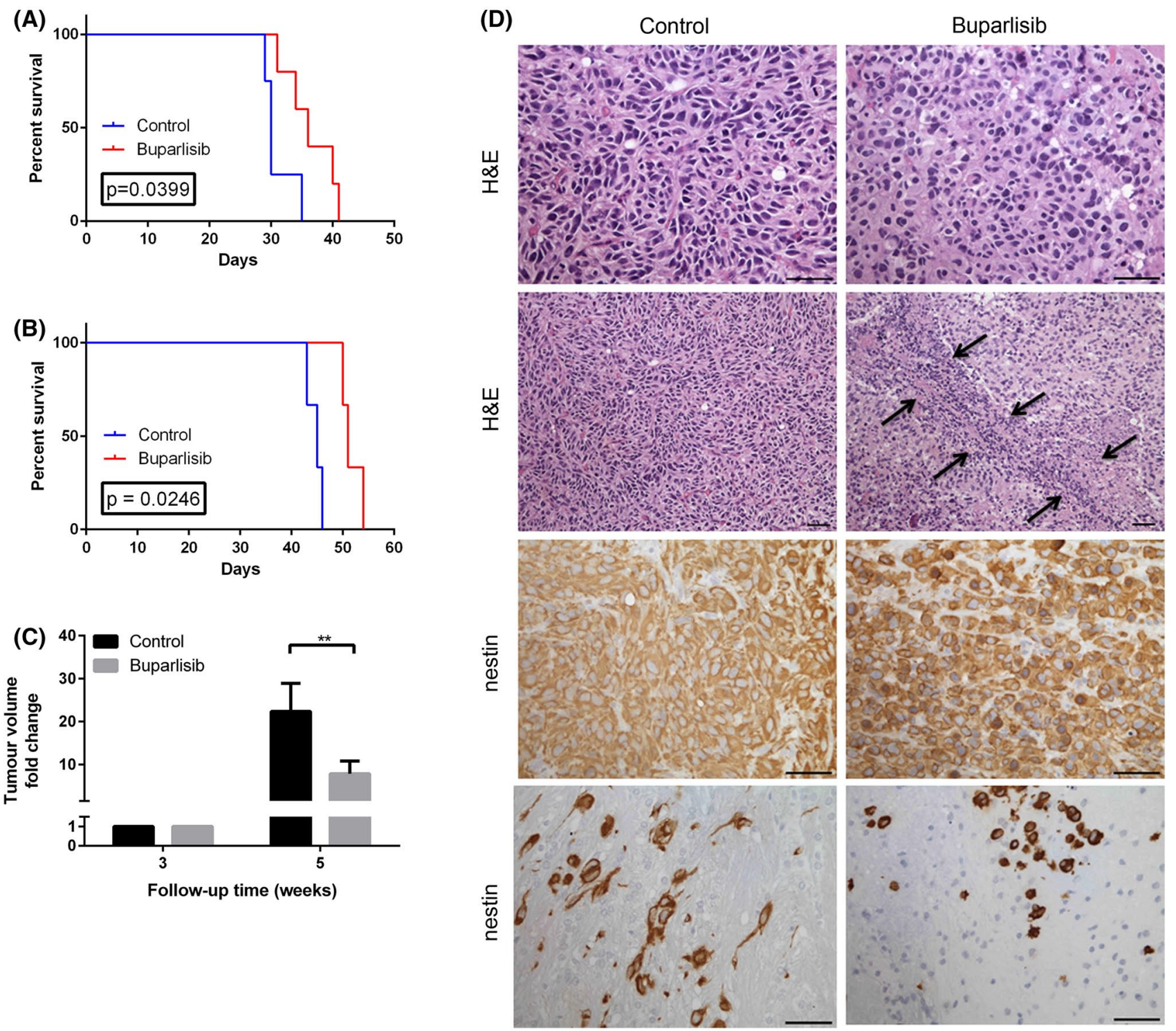

Fig. 3 a Kaplan-Meyer survival curve for first study with nude rats carrying P3 xenografts $(n=9)$. b Kaplan-Meyer survival curve for the second study, with nude rats carrying P3 xenografts $(n=6)$. c MRIbased assessments of tumour volumes 3 weeks post implantation (treatment start) and 5 weeks post implantation. $* \mathrm{p}<0.05$. $\mathbf{d}$ H\&E and nestin immunostained sections of treated and control tumors. Arrows indicate a necrotic tumour area. Scale bars $50 \mu \mathrm{m}$ cell nuclei seemed to be reduced in the treated compared to control tumours. As the intermediate filament protein nestin is expressed in a very high fraction of tumour cells, we used it as a tumour marker. Immunostaining for nestin revealed that the morphology of tumour cells changed in the treated animals compared to controls. Tumour cells from the treated group showed a more epithelial-like phenotype while the tumour cells of the control tumours had a mesenchymal shape which was best visible in infiltrative tumour areas (Fig. 3d).

To confirm previous findings in a larger study we stereotactically implanted U87 glioma spheroids and initiated buparlisib treatment after tumour engraftment was verified by MRI (10 days post implantation). In this study, the median survival in the control group $(n=9)$ was 25.5 days (range 19-39 days) from implantation versus 30 days (range 24-67 days) in buparlisib-treated rats $(n=9)(p=0.0097$, Fig. 4a). Notably, in the treatment group, some animals lived several weeks longer than untreated animals. MRI scans performed weekly revealed stable tumour volume, indicating prolonged progression free survival in the animals responding to the therapy (Fig. 4c). However, after the initial response, the tumours resumed growth and the animals developed symptoms. 
(A)

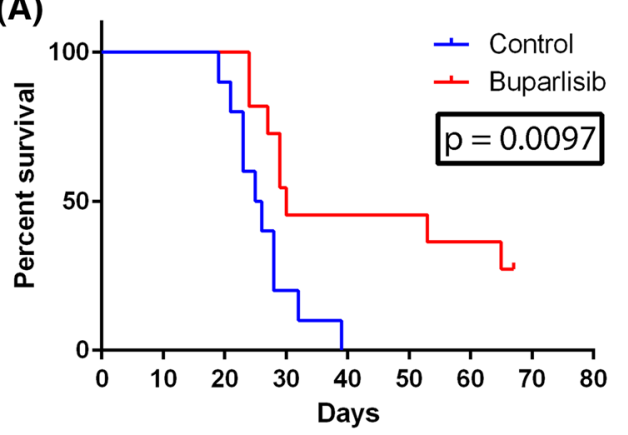

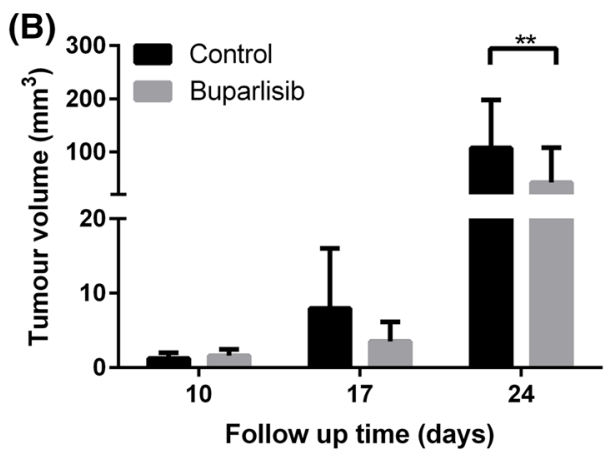

(C)

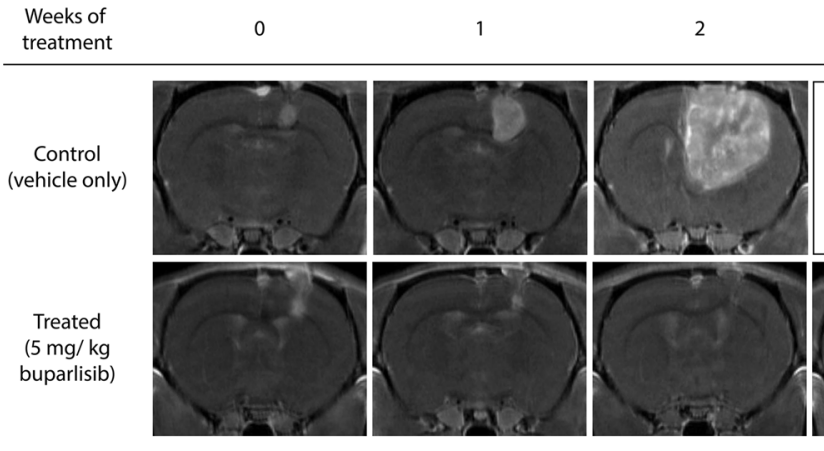

Fig. 4 a Kaplan-Meyer survival curve for nude rats carrying orthotopic GBM cell line (U87) xenografts $(n=18)$. b MRI-based assessments of tumour volumes 10 days post implantation (treatment start)

\section{Buparlisib inhibits phosphorylation of Akt in vivo}

Ex vivo western blot analysis of tumour tissues from euthanized rats showed significant inhibition of Akt phosphorylation at S473. Phosphorylation at T308 was also reduced following treatment, although this difference was not significant. The total level of Akt protein was unchanged (Fig. 5a, b).

\section{Daily treatment with buparlisib in nude rats harbouring GBM xenografts is well tolerated}

Daily inspection of the rats showed no change of activity or food and water intake. Throughout the experiment, both the treated and control animals showed stable body weight, although the treatment group displayed a slight weight reduction (Fig. $5 \mathrm{c}$ ). The rats in the treatment group had temporary hair loss after 3 weeks of treatment. However, the animals exhibited hair re-growth while they were still on treatment. No severe side effects were observed.

\section{Discussion}

We evaluated the anti-tumour efficacy of pan-PI3K inhibitor buparlisib on glioma. A dose dependent anti-proliferative effect of buparlisib in vitro, accompanied by inhibition and 24 days post implantation. ${ }^{*} \mathrm{p}<0.05$ c $\mathrm{T} 1$-weighted magnetic resonance images (MRI) with contrast, from two representative rats; one from each group

of Akt phosphorylation at both serine 473 (S473) and threonine 308 (T308) was demonstrated. In vivo, buparlisib treatment led to significantly improved survival and reduced tumour volume. The compound seemed to be well tolerated by the animals, also during prolonged treatment over several weeks.

The observed in vitro anti-proliferative effect of buparlisib confirms previous findings, which include cell lines of glioma origin [9, 12], as well as other cell lines [9]. The ability of buparlisib to induce apoptosis as well as dose dependent reduction of Akt phosphorylation in vitro both at $\mathrm{S} 473$ and T308 is in line with previous reports $[9,13]$.

In our study, buparlisib demonstrated anti-tumour efficacy in an animal model employing patient-derived tumour material that was previously shown to mimic the growth of human gliomas in situ [14]. The three independent animal experiments confirmed previous reports of prolonged survival of animals with intracranial GBM xenografts [9]. However the efficacy of buparlisib in GBM therapy has not previously been studied using in vivo propagated patientderived tumour material. Our results were further validated with a commonly used glioma cell line U87 [15]. However, we initiated buparlisib treatment up to 3 weeks following tumour implantation, after tumour engraftment was confirmed by MRI. Although, Koul et al. reported growth inhibition in an in vivo GBM model using buparlisib [9], they 
(A) Ctrl Buparlisib

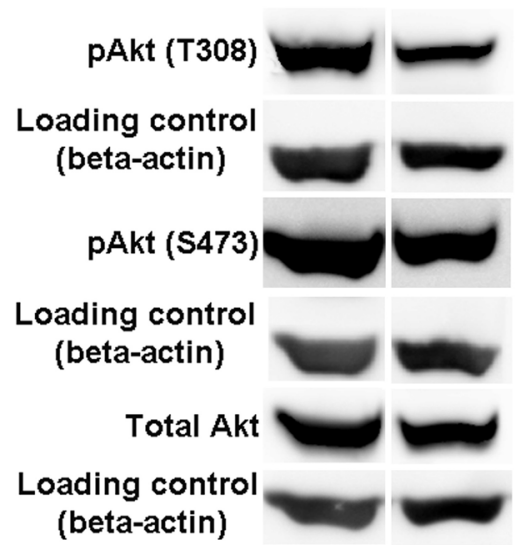

(B)

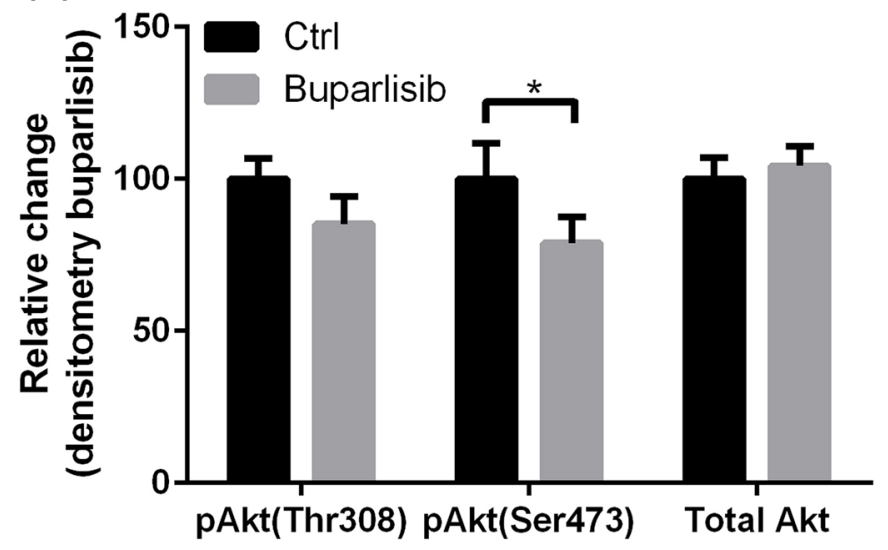

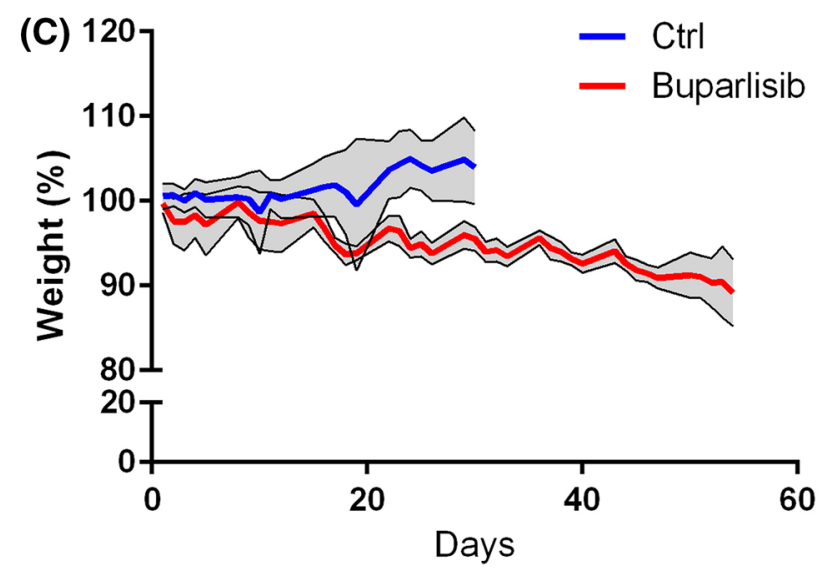

Fig. 5 a Western blots showing levels of pAkt (T308), pAkt (S473) and total Akt in the tumours of one representative U87-xenografted rats from each group. Tumor material was collected $2-4 \mathrm{~h}$ post treatment when rats had reached humane endpoints. b Densitometric assessment of the western blot in (a), showing relative change in phosphorylation. $* \mathrm{p}<0.05$. $\mathbf{c}$ Weight measurements for the two groups initiated treatment shortly after tumour implantation without prior confirmation of tumour engraftment. We believe our present data obtained in a model closely resembling the clinical setting where the relapsed tumour is detected by MRI, provide additional support for clinical validation of buparlisib for human GBMs. Interestingly, the observed anti-tumour efficacy of buparlisib extends beyond previous results, as one third of the animals experienced prolonged progression free survival and even slight reduction in tumour size for several weeks. However, the effect was temporary as the tumours eventually resumed growth. This reflects the palliative therapy of solid tumours, when tumour progression occurs after initial volume response and/or disease stabilization. Bradford et al. have also reported development of secondary resistance to buparlisib therapy. In endometrial cancer the resistance was mitigated by conventional chemotherapy [16].

Ex vivo analysis of the tumour samples obtained post mortem from treated animals demonstrated decreased phosphorylation of Akt, confirming that buparlisib does reach its intracranial target. This is in line with the published study of Koul and colleagues [9] and the previously reported BBB penetrance [9]. The histopathology of the tumours was altered in the treated animals indicating the therapeutic effect of the compound. Moreover, buparlisib seemed to be well tolerated by the animals, also over a prolonged exposure time. Similar observations have been made in phase I/ II clinical studies of buparlisib [17-19].

Treatment resistance in cancer is commonly mediated through activation of the PI3K pathway [20]. Targeting PI3K may thus be a plausible strategy for treatment of GBM. Previous studies demonstrated that inhibition of PI3K increases the sensibility of GBM cells to doxorubicin treatment in vitro [21]. Buparlisib has demonstrated an additive effect when combined with temozolomide in glioma cells, and a synergistic effect when combined with MEK and HER2 inhibitors [9]. Here we show anti-tumour efficacy of a PI3K inhibitor alone. The secondary resistance to PI3K inhibitor might be attenuated by combining buparlisib with conventional chemotherapy. Currently, there are ongoing clinical 
trials assessing buparlisib monotherapy in recurrent GBM as well as of combination of buparlisib with other agents in newly diagnosed and recurrent malignant glioma.

In conclusion, we have shown that buparlisib has antitumour efficacy as a monotherapy in preclinical studies. Thus, we believe our data provide a rationale for clinical trials validating buparlisib as a single therapy in GBM patients.

Acknowledgments The MR-imaging was performed at the Molecular Imaging Center (MIC) and was thus supported by the Department of Biomedicine and the Faculty of Medicine and Dentistry, at the University of Bergen, and its partners. Financial support was received from the University of Bergen (UiB), Helse Vest and Norwegian cancer society (Kreftforeningen) and Kristian Gerhard Jebsen Brain Tumour Research Center. In addition, Dorota Goplen received financial support from Novartis to perform the animal experiments, including the drug buparlisib as a kind gift.

Author contribution IAN and HEF designed all conducted experiments and coordinated the study. They did most of the work with animal studies in addition to central parts of the in vitro experiments and analysis. LS and BSS helped with animal experiments, LL and MAR performed some parts of the in vitro experiments. HM analysed histology sections. Advisors PØE and DG gave a lot of helpful suggestions and feedback to the ongoing experiments and writing. All authors read and approved the manuscript.

\section{Compliance with ethical standards}

Conflict of interest The authors declare that they have no conflict of interests.

Open Access This article is distributed under the terms of the Creative Commons Attribution 4.0 International License (http://creativecommons.org/licenses/by/4.0/), which permits unrestricted use, distribution, and reproduction in any medium, provided you give appropriate credit to the original author(s) and the source, provide a link to the Creative Commons license, and indicate if changes were made.

\section{References}

1. Louis DN et al (2007) The 2007 WHO classification of tumours of the central nervous system. Acta Neuropathol 114(2):97-109

2. Stupp R et al (2009) Effects of radiotherapy with concomitant and adjuvant temozolomide versus radiotherapy alone on survival in glioblastoma in a randomised phase III study: 5-year analysis of the EORTC-NCIC trial. Lancet Oncol 10(5):459-466

3. Fruman DA, Rommel C (2014) PI3K and cancer: lessons, challenges and opportunities. Nat Rev Drug Discov 13(2):140-156
4. Akinleye A et al (2013) Phosphatidylinositol 3-kinase (PI3K) inhibitors as cancer therapeutics. J Hematol Oncol 6(1):88

5. Wen PY et al (2012) Current clinical development of PI3K pathway inhibitors in glioblastoma. Neuro Oncol 14(7):819-829

6. Brennan CW et al (2013) The somatic genomic landscape of glioblastoma. Cell 155(2):462-477

7. Chakravarti A et al (2004) The prognostic significance of phosphatidylinositol 3-kinase pathway activation in human gliomas. $\mathbf{J}$ Clin Oncol 22(10):1926-1933

8. Sarbassov DD et al (2005) Phosphorylation and regulation of Akt/ PKB by the rictor-mTOR complex. Science 307(5712):1098-1101

9. Koul D et al (2012) Antitumor activity of NVP-BKM120a selective pan class I PI3 kinase inhibitor showed differential forms of cell death based on p53 status of glioma cells. Clin Cancer Res 18(1):184-195

10. Wang J et al (2009) A reproducible brain tumour model established from human glioblastoma biopsies. BMC Cancer 9:465

11. Bjerkvig R et al (1990) Multicellular tumor spheroids from human gliomas maintained in organ culture. J Neurosurg 72(3):463-475

12. Jane EP et al (2014) Inhibition of phosphatidylinositol 3-kinase/ AKT signaling by NVP-BKM120 promotes ABT-737-induced toxicity in a caspase-dependent manner through mitochondrial dysfunction and DNA damage response in established and primary cultured glioblastoma cells. J Pharmacol Exp Ther 350(1):22-35

13. Sanchez CG et al (2011) Preclinical modeling of combined phosphatidylinositol-3-kinase inhibition with endocrine therapy for estrogen receptor-positive breast cancer. Breast Cancer Res 13(2):R21

14. Kerbel RS (2003) Human tumor xenografts as predictive preclinical models for anticancer drug activity in humans: better than commonly perceived-but they can be improved. Cancer Biol Ther 2(4 Suppl 1):S134-S139

15. Jacobs VL et al (2011) Current review of in vivo GBM rodent models: emphasis on the CNS-1 tumour model. ASN Neuro 3(3): 00063

16. Bradford LS et al (2014) Assessing the efficacy of targeting the phosphatidylinositol 3-kinase/AKT/mTOR signaling pathway in endometrial cancer. Gynecol Oncol 133(2):346-352

17. Rodon $J$ et al (2014) Phase I dose-escalation and expansion study of buparlisib (BKM120), an oral pan-class I PI3K inhibitor, in patients with advanced solid tumors. Invest New Drugs 32(4):670-681

18. Ando $\mathrm{Y}$ et al (2014) Phase I dose-escalation study of buparlisib (BKM120), an oral pan-class I PI3K inhibitor, in Japanese patients with advanced solid tumors. Cancer Sci 105(3):347-353

19. Bendell JC et al (2012) Phase I, dose-escalation study of BKM120, an oral pan-class I PI3K inhibitor, in patients with advanced solid tumors. J Clin Oncol 30(3):282-290

20. Burris HA 3rd (2013) Overcoming acquired resistance to anticancer therapy: focus on the PI3K/AKT/mTOR pathway. Cancer Chemother Pharmacol 71(4):829-842

21. Johannessen TC et al (2009) Highly infiltrative brain tumours show reduced chemosensitivity associated with a stem cell-like phenotype. Neuropathol Appl Neurobiol 35(4):380-393 